\title{
Characterization of Changes in 'Gala' Apple Aroma during Storage Using Osme Analysis, a Gas Chromatography-Olfactometry Technique
}

\author{
Anne Plotto ${ }^{1}$ and Mina R. McDaniel ${ }^{2}$ \\ Department of Food Science and Technology, Oregon State University, Corvallis, OR 97331 \\ James P. Mattheis ${ }^{3}$ \\ U.S. Department of Agriculture, Tree Fruit Research Center, 1104 N. Western Avenue, Wenatchee, WA 98801
}

\begin{abstract}
AdDitional INDEX words. Malus $\times$ sylvestris var. domestica, fruit, volatiles, controlled atmosphere, esters, odor-active, headspace

Abstract. Changes in the odor-active volatile compounds produced by 'Gala' apples [Malus $\times$ sylvestris (L.) Mill. var. domestica (Borkh.) Mansf. 'Gala'] were measured after 4, 10, and 20 weeks storage at $1{ }^{\circ} \mathrm{C}$ in regular atmosphere (RA) or controlled atmosphere (CA), and 16 weeks in CA followed by 4 weeks in RA. Aroma was evaluated using the gas chromatography-olfactometry method $O$ sme. Production of volatile esters decreased along with corresponding fruity aromas during CA storage. Hexyl acetate, butyl acetate, and 2-methylbutyl acetate were emitted in the largest amounts and perceived with the strongest intensities from RA-stored fruit. While hexyl acetate and butyl acetate concentrations and aroma intensities decreased during $\mathrm{CA}$ storage, 2-methylbutyl acetate remained at the $\mathrm{RA}$ concentration until apples had been stored 16 weeks in CA. Perception intensities of methylbutyrate esters with apple or berrylike odors decreased less than straight chain esters in CA-stored fruit. 4-Allylanisole, B-damascenone, and 1-octen-3-ol, as well as an unknown compound with a watermelon descriptor, were perceived more in RA-stored fruit than in CA-stored apples. Factor analysis indicated the importance of these compounds in 'Gala' apples stored 4 weeks in RA. Even though these compounds do not have an apple odor, they contribute to fresh 'Gala' aroma.
\end{abstract}

Sniffing effluents of a gas chromatograph to determine odor characteristics of volatile compounds present in a sample has been practiced since the beginning of gas chromatography. Information relative to odor character of compounds found in apples and determined by gas chromatography and olfactometry (GCO) was first published by Guadagni et al. (1966). GCO has been used to describe odorous compounds emitted by intact 'Golden Delicious' apples (Malus sylvestris var. domestica) (Pérez et al., 1993; Rizzolo et al., 1992; Rizzolo et al., 1989), and compounds extracted from 'Kogyoku' apples by steam distillation (Yajima et al., 1984). CharmAnalysis, a GCO technique that determines the potency of odor-active peaks, has been used to investigate differences among apple cultivars (Cunningham et al., 1986), as well as to characterize compounds contributing to 'Gala' aroma (Young et al., 1996). Young and coworkers found 2-methylbutyl acetate, butyl acetate, hexyl acetate, and butanol to be important contributors to 'Gala' aroma.

All the above mentioned GCO studies reported only odor qualities for the compounds found in apples, and no comparisons were made between storage treatments or maturity stages. 'Gala' apple originated in New Zealand and has gained worldwide popularity because of its unique flavor (White, 1991). However, the storage season for 'Gala' is short, in part due to a decrease in aroma quality after storage (Plotto et al., 1995; Stebbins et al.,

Received for publication 19 July 1999. Accepted for publication 20 June 2000. Joint publication of the Oregon Agricultural Experiment Station and the U.S. Department of Agriculture, Agricultural Research Service. This research was funded in part by the Washington State Tree Fruit Research Commission and the Oregon Agricultural Experiment Station. We acknowledge Thomas D. Mann for statistical support of this research. The cost of publishing this paper was defrayed in part by the payment of page charges. Under postal regulations, this paper therefore must be hereby marked advertisement solely to indicate this fact.

${ }^{1}$ Graduate research assistant.

${ }^{2}$ Professor.

${ }^{3}$ Research plant physiologist
1994). Controlled atmosphere storage (CA) using reduced $\mathrm{O}_{2}$ and increased $\mathrm{CO}_{2}$ concentrations minimizes apple fruit firmness, acidity, and chlorophyll losses and delays the appearance of some storage disorders (Smock, 1979). However, reduced production of volatile compounds is also observed following CA storage of apples (Patterson et al., 1974; Streif and Bangerth, 1988; Yahia et al., 1990). Apple maturity stage at harvest (Mattheis et al., 1991; Song and Bangerth, 1996), ratio of $\mathrm{O}_{2}$ and $\mathrm{CO}_{2}$ in the atmosphere (Brackmann et al., 1993; Fellman et al., 1993; Streif and Bangerth, 1988) as well as storage duration (Willaert et al., 1983) affect the recovery of volatile production after CA storage.

The objectives of the present study were to further identify volatile compounds contributing to the aroma of 'Gala' apples, and to evaluate the impact of CA storage on aroma production. The GCO technique Osme was used to measure the response of panelists to odorants. In Osme analysis, panelists record the intensity and odor descriptor of perceived compounds from an undiluted food extract (Da Silva et al., 1994; McDaniel et al., 1990). Using trained panelists to record intensities of perceived compounds in multiple evaluations, Osme data are reproducible (Da Silva et al., 1994) and can be analyzed with parametric statistical techniques (Da Silva et al., 1993). Results of Osme analyses were used to interpret the changes of 'Gala' flavor in storage by descriptive sensory analysis (DSA) (Plotto et al., 1999).

\section{Materials and Methods}

Plant material and storage. 'Gala' apples were harvested in a commercial orchard near Chelan, Wash., on 12 Sept. 1995. Apple maturity stage and homogeneity of the lots were assessed by ground color, internal ethylene concentration (IEC), and starch score (Plotto et al., 1995). Twenty fruit were held for $24 \mathrm{~h}$ at $20^{\circ} \mathrm{C}$ before analysis of internal ethylene concentration (IEC) 
and starch score. Gas samples taken from the fruit core were analyzed to determine IEC (Williams and Patterson, 1962). The analyses were conducted isothermally at $50{ }^{\circ} \mathrm{C}$ using a gas chromatograph (GC) (HP 5880A, Agilent Technologies, Palo Alto, Calif.) equipped with a $50 \mathrm{~cm}, 0.32 \mathrm{~cm}$ i.d. glass column packed with 80 to 100 mesh Porapak Q (Supelco, Bellefonte, Pa.). Nitrogen carrier, $\mathrm{H}_{2}$, and air flows were 25,25 , and $300 \mathrm{~mL} \cdot \mathrm{min}^{-1}$, respectively. Starch score was rated visually using a 1 to 6 scale ( $1=$ full starch and $6=$ no starch) after staining an equatorial section with a $0.5 \% \mathrm{w} / \mathrm{v}$ ) I-KI solution.

Fruit were stored at the USDA-ARS Tree Fruit Research Laboratory, Wenatchee, Wash., at $1{ }^{\circ} \mathrm{C}$ for 4,10 or 20 weeks in regular atmosphere (RA), in controlled atmosphere $(\mathrm{CA})$ at $1 \mathrm{kPa}$ $\mathrm{O}_{2}$ and $1 \mathrm{kPa} \mathrm{CO}$, or for 16 weeks in CA followed by 4 weeks in RA. After removal from storage, fruit were shipped to Corvallis, Ore., stored at $2^{\circ} \mathrm{C}$ for $5 \mathrm{~d}$, and then ripened at $22^{\circ} \mathrm{C}$ for $5 \mathrm{~d}$ before volatile collection.

Headspace Sampling. Four replicate samples (five apples each, $\approx 1 \mathrm{~kg}$ ) were placed in 4-L glass jars sealed using Teflon lids with two gas ports. Compressed air purified by flowing through activated charcoal, calcium hydroxide, and $0.5 \mathrm{~nm}$ molecular sieve(W.A. Hammond Drierite, Xenia, Ohio) was passed through the jars at $\approx 200 \mathrm{~mL} \cdot \mathrm{min}^{-1}$. Volatiles were collected on activated coconut charcoal, 20/40 mesh, $150 \mathrm{mg}$, ORBO-32 (Supelco) for $24 \mathrm{~h}$. The choice of traps for sampling of volatile compounds, as well as sampling time were optimized for this specific study (Plotto, 1998).

Sampling took place in a ripening chamber maintained at 22 ${ }^{\circ} \mathrm{C}$. Traps were stored at $-25^{\circ} \mathrm{C}$ until elution. Volatile compounds were desorbed from charcoal in $1.8-\mathrm{mL}$ vials with $300 \mu \mathrm{L} \mathrm{CS}_{2}$ (HPLC grade, 99.9\%+, Sigma-Aldrich, St. Louis, Mo.) containing tridecane at $100 \mathrm{mg} \cdot \mathrm{L}^{-1}$ (Sigma-Aldrich) as an internal standard. Samples (sorbent and solvent) were stored at $-17^{\circ} \mathrm{C}$ before analysis.

Gas Chromatography-olfactometry. Samples were analyzed using a GC (HP 5890, Agilent Technologies) gas chromatograph equipped with a three-way valve (Valco Instruments Co., Inc., Houston, Texas) to direct the column flow to a flame-ionization detector (FID) or a sniff port. The analytical column was a Rtx-5 fused silica column coated with crossbond 5\% diphenyl-95\% dimethyl polysiloxane, $30 \mathrm{~m}, 0.53 \mathrm{~mm}$ i.d., and $1-\mu \mathrm{m}$ film thickness (Restek, Bellefonte, Pa.). Conditions for GC were as follows: splitless injection at $250^{\circ} \mathrm{C}$, initial oven temperature $40{ }^{\circ} \mathrm{C}$ held for $1 \mathrm{~min}$, increased to $165^{\circ} \mathrm{C}$ at $5^{\circ} \mathrm{C} \cdot \mathrm{min}^{-1}$, to $250^{\circ} \mathrm{C}$ at $20^{\circ} \mathrm{C} \cdot \mathrm{min}^{-1}$, then held for $15 \mathrm{~min}$. The FID temperature was $280^{\circ} \mathrm{C}$ and flow rates for $\mathrm{H}_{2}$, air, and auxiliary gas (He) to the FID were 30,390 , and $27 \mathrm{~mL} \cdot \mathrm{min}^{-1}$, respectively. Linear velocity of He carrier gas was $30.7 \mathrm{~cm} \cdot \mathrm{s}^{-1}$. The sniff port was a $40 \mathrm{~cm}$ long, $4 \mathrm{~mm}$ diameter glass tube deactivated with $5 \%$ dimethyldichlorosilane (Sylon-CT, Supelco) connected with a tee to the outlet of the GC column. Compressed air (breathing quality) flowing to the sniff port at $3.5 \mathrm{~L} \cdot \mathrm{min}^{-1}$ was purified and humidified through activated charcoal, $0.5 \mathrm{~nm}$ molecular sieve, and $2 \mathrm{~L}$ distilled water held at $30^{\circ} \mathrm{C}$. Three panelists were trained to smell and describe the column effluents while rating the perceived intensity on a 16 -point intensity scale $(0=$ none and 15 = extreme). Intensity was recorded by moving a linear sliding bar connected to a variable resistor interfaced to a personal computer (Da Silva et al., 1994). Headspace samples collected from 'Gala' apples were used for training. Panelists were asked to identify the strongest odor peak and scale the intensity of the rest of the aromagram as to how intense they perceived the peaks. After the panelists had been familiarized with the sample and had developed their own vocabulary, reference standards were provided before each sniffing session so that panelists remained consistent in the naming of odors and assigning intensities. Each sniffing session started after the solvent had completely eluted from the column and lasted until all odor-active compounds had eluted, $\approx 30 \mathrm{~min}$. Data were recorded for time duration and intensity for individual compounds with Osme v.1.0 for Windows 3.1, (Oregon State Univ., Corvallis). The resulting output for each response was a) duration of odor perception, b) maximum odor intensity $\left(I_{\max }\right)$, c) area under the curve generated by the odor stimulus response (duration $\times$ intensity), and $d$ ) retention index at the time of maximum perceived intensity. Retention indices were calculated after GC analysis of a series of hydrocarbon standards under the same conditions as the volatile samples.

Compounds were identified initially by GC-mass spectrometry (MS). Samples were analyzed with a GC (HP 5890 series II, Agilent Technologies) equipped with a MS detector (HP 5971a, Agilent Technologies) and a DB-5, $30 \mathrm{~m}, 0.25 \mathrm{~mm}$ i.d., $0.25-\mu \mathrm{m}$ film thickness capillary column (J\&W Scientific, Folsom, Calif.). The temperature program and other chromatography conditions were identical to those stated previously. Spectra of individual compounds were compared with those in the Wiley/NBS database (1988). Confirmation of identification was made by 1) comparing retention indices of authentic standards (Aldrich Flavors and Fragrances, Milwaukee, Wis.) and 2) Osme evaluation of those standards in the same quantities as in the sample. When the odor of a sample compound differed from that of its putative authentic standard, the compound was not included in further analysis, even though the standard and sample volatiles exhibited similar mass spectral characteristics and retention indices. All standards used for olfactometry were food grade. Quantification of identified compounds was calculated from calibration curves of authentic standards run on the GC-FID.

STATISTICAL DESIGN AND ANALYSES. All statistical analyses were performed using SAS statistical software v.6.12 (SAS Inst., Inc., Cary, N.C.), and model terminology was according to SAS (1987). Unless otherwise noted, only results significant at $P \leq$ 0.05 are discussed.

All panelists evaluated each of the four replicate samples once for each storage treatment. Samples were presented in a completely randomized order blocked by sample replication. For statistical analysis, storage type (RA or CA) and duration were treated independently and are hereafter referred to as "storage treatment". Differences between storage treatments were analyzed for each volatile compound using a one-way analysis of variance (ANOVA), with storage as the main effect.

For each set of peak odor intensity $\left(I_{\max }\right)$ or peak odor area response variables, differences between storage treatments were analyzed using a four-way ANOVA. Sample replicate was nested within storage treatment [Sample(Storage)]. The model was Response variable $=$ Storage sample(Storage) Panelist Panelist $\times$ Storage. Storage and Panelist were treated as fixed effects while Sample and therefore Sample(Storage) was treated as a random effect. Sample(Storage) was used as the error term for the F ratio; therefore, apple variability was tested and panelist was included in the experimental error.

Data were also examined using multivariate analysis. Factor analysis (FA) was chosen as an exploratory technique to describe and summarize data by grouping together variables that are correlated (Tabachnick and Fidel, 1989). Variables were: identified odor-active volatile compounds, peak intensities $\left(I_{\max }\right)$, and 
Table 1. Volatile compounds emitted by 'Gala' apples after regular (RA) or controlled atmosphere $(\mathrm{CA})$ storage $\left(1 \mathrm{kPa} \mathrm{O}_{2}, 1 \mathrm{kPa} \mathrm{CO}_{2}\right)$. Values $\left(\mathrm{ng} \cdot \mathrm{kg}^{-1} \cdot \mathrm{L}^{-1}\right)$ are means of four replicates collected using dynamic headspace sampling of $1 \mathrm{~kg}$ apples. Total volatiles by chemical group are also presented.

\begin{tabular}{|c|c|c|c|c|c|c|}
\hline \multirow[b]{2}{*}{ Compound } & \multicolumn{3}{|c|}{ RA storage (weeks) } & \multicolumn{3}{|c|}{ CA storage (weeks) } \\
\hline & 4 & 10 & 20 & 10 & $16^{2}$ & 20 \\
\hline Propyl acetate & $409 \mathrm{a}^{\mathrm{y}}$ & $261 \mathrm{a}$ & $271 \mathrm{a}$ & $54 \mathrm{~b}$ & $27 \mathrm{~b}$ & $3 \mathrm{~b}$ \\
\hline Butyl acetate ${ }^{\mathrm{x}, \mathrm{w}}$ & 3860 a & $4000 \mathrm{a}$ & $4260 \mathrm{a}$ & $1980 \mathrm{~b}$ & $610 \mathrm{bc}$ & $72 \mathrm{c}$ \\
\hline Pentyl acetate ${ }^{\mathrm{x}}$ & $353 \mathrm{a}$ & $193 \mathrm{bc}$ & $262 \mathrm{ab}$ & $128 \mathrm{bcd}$ & $45 \mathrm{~cd}$ & $11 \mathrm{~d}$ \\
\hline Hexyl acetate ${ }^{\mathrm{x}, \mathrm{w}}$ & $2930 \mathrm{a}$ & $2530 \mathrm{ab}$ & $2725 \mathrm{ab}$ & $1760 \mathrm{~b}$ & $663 c$ & $142 \mathrm{c}$ \\
\hline Heptyl acetate & $66 \mathrm{a}$ & $23 \mathrm{~b}$ & $24 \mathrm{~b}$ & $23 \mathrm{~b}$ & $9 \mathrm{bc}$ & $3 c$ \\
\hline Total acetate esters & 7618 & 7007 & 7542 & 3945 & 1354 & 230 \\
\hline Propyl propanoate ${ }^{\mathrm{x}} 34 \mathrm{a}$ & $18 \mathrm{~b}$ & $14 \mathrm{bc}$ & $4 \mathrm{bc}$ & $3 c$ & $1 \mathrm{c}$ & \\
\hline Butyl propanoate $^{\mathrm{x}}$ & $691 \mathrm{a}$ & 429 a & $534 \mathrm{a}$ & $100 \mathrm{~b}$ & $41 \mathrm{~b}$ & $4 \mathrm{~b}$ \\
\hline Pentyl propanoate & $22 \mathrm{a}$ & $9 \mathrm{bc}$ & $14 \mathrm{~b}$ & $11 \mathrm{bc}$ & $5 \mathrm{~cd}$ & $1 \mathrm{~d}$ \\
\hline Hexyl propanoate ${ }^{\mathrm{x}}$ & $537 \mathrm{a}$ & $264 \mathrm{bc}$ & $333 \mathrm{~b}$ & $126 \mathrm{~cd}$ & $58 \mathrm{~d}$ & $9 \mathrm{~d}$ \\
\hline Total propanoate esters & 1284 & 720 & 895 & 241 & 107 & 15 \\
\hline Propyl butyrate & $40 \mathrm{a}$ & $17 \mathrm{bc}$ & $23 \mathrm{~b}$ & $10 \mathrm{bcd}$ & $8 \mathrm{~cd}$ & $1 \mathrm{~d}$ \\
\hline Butyl butyrate & $536 \mathrm{a}$ & $256 \mathrm{~b}$ & $484 \mathrm{a}$ & $153 \mathrm{bc}$ & $59 \mathrm{c}$ & $12 \mathrm{c}$ \\
\hline Pentyl butyrate & $21 \mathrm{a}$ & $11 \mathrm{bc}$ & $15 \mathrm{abc}$ & $24 \mathrm{a}$ & $18 \mathrm{ab}$ & $7 c$ \\
\hline Hexyl butyrate $^{x}$ & $429 a b$ & $328 \mathrm{~b}$ & 569 a & $255 \mathrm{bc}$ & $109 \mathrm{~cd}$ & $29 d$ \\
\hline Total butyrate esters & 1025 & 612 & 1090 & 442 & 194 & 49 \\
\hline Propyl hexanoate & $179 \mathrm{a}$ & $41 \mathrm{bc}$ & $54 \mathrm{~b}$ & $27 \mathrm{bc}$ & $16 \mathrm{bc}$ & $5 \mathrm{c}$ \\
\hline Butyl hexanoate $^{x}$ & $1310 \mathrm{a}$ & $632 \mathrm{~b}$ & $1060 \mathrm{a}$ & $527 \mathrm{bc}$ & $236 \mathrm{~cd}$ & $64 \mathrm{~d}$ \\
\hline Hexyl hexanoate & $1610 \mathrm{a}$ & $277 \mathrm{c}$ & $501 \mathrm{~b}$ & $214 \mathrm{~cd}$ & $108 \mathrm{~cd}$ & $33 \mathrm{~d}$ \\
\hline Total hexanoate esters & 3099 & 950 & 1615 & 768 & 360 & 101 \\
\hline Butyl heptanoate & $135 \mathrm{a}$ & $27 \mathrm{bc}$ & $43 \mathrm{~b}$ & $31 \mathrm{bc}$ & $15 \mathrm{bc}$ & $5 \mathrm{c}$ \\
\hline Hexyl octanoate & $63 \mathrm{a}$ & $15 \mathrm{bc}$ & $21 \mathrm{~b}$ & $10 \mathrm{bcd}$ & $5 \mathrm{~cd}$ & $1 \mathrm{~d}$ \\
\hline 2-Methylpropyl acetate & $99 \mathrm{~b}$ & $76 \mathrm{bc}$ & $173 \mathrm{a}$ & $111 \mathrm{~b}$ & $72 \mathrm{bc}$ & $26 \mathrm{c}$ \\
\hline 2-Methylbutyl acetate ${ }^{\mathrm{x}, \mathrm{w}}$ & $2100 \mathrm{a}$ & $1090 \mathrm{bc}$ & $1460 \mathrm{ab}$ & $1970 \mathrm{a}$ & $1430 \mathrm{ab}$ & $438 \mathrm{c}$ \\
\hline 3-Methyl-2-butenyl acetate ${ }^{\mathrm{w}}$ & $45 \mathrm{~b}$ & $10 \mathrm{c}$ & $9 \mathrm{c}$ & $110 \mathrm{a}$ & $122 \mathrm{a}$ & $40 \mathrm{~b}$ \\
\hline 3-Methylbutyl propanoate & $26 \mathrm{a}$ & $7 \mathrm{~cd}$ & $7 \mathrm{~cd}$ & $20 \mathrm{ab}$ & $14 \mathrm{bc}$ & $3 \mathrm{~d}$ \\
\hline 2-Methylbutyl butyrate & $9 a b$ & $5 \mathrm{bc}$ & $7 \mathrm{ab}$ & $10 \mathrm{a}$ & $8 \mathrm{ab}$ & $3 c$ \\
\hline 3-Methylbutyl hexanoate & $23 \mathrm{a}$ & $5 \mathrm{c}$ & $7 \mathrm{c}$ & $16 \mathrm{~b}$ & $15 \mathrm{~b}$ & $5 \mathrm{c}$ \\
\hline Butyl 2-methylpropanoate & $14 \mathrm{~b}$ & $12 \mathrm{~b}$ & $23 \mathrm{a}$ & $6 \mathrm{bc}$ & $3 \mathrm{c}$ & $1 \mathrm{c}$ \\
\hline Hexyl 2-methylpropanoate & $25 \mathrm{ab}$ & $19 \mathrm{bc}$ & $41 \mathrm{a}$ & $15 \mathrm{bcd}$ & $7 \mathrm{~cd}$ & $1 \mathrm{~d}$ \\
\hline Total methyl propanoate esters & 39 & 31 & 64 & 21 & 10 & 2 \\
\hline Methyl 2-methylbutyrate ${ }^{\mathrm{x}}$ & $60 \mathrm{~b}$ & $46 \mathrm{bc}$ & $100 \mathrm{a}$ & $11 \mathrm{~d}$ & $19 \mathrm{~cd}$ & $0 \mathrm{~d}$ \\
\hline Ethyl 2-methylbutyrate & $5 \mathrm{ab}$ & $3 \mathrm{bc}$ & $8 \mathrm{a}$ & $1 \mathrm{c}$ & $1 \mathrm{c}$ & $0 \mathrm{c}$ \\
\hline Propyl 2-methylbutyrate ${ }^{\mathrm{x}}$ & $114 \mathrm{a}$ & $56 \mathrm{~b}$ & $46 \mathrm{bc}$ & $12 \mathrm{bcd}$ & $10 \mathrm{~cd}$ & $1 \mathrm{~d}$ \\
\hline Butyl 2-methylbutyrate ${ }^{x}$ & $724 \mathrm{a}$ & $528 \mathrm{a}$ & $730 \mathrm{a}$ & $158 \mathrm{~b}$ & $62 \mathrm{~b}$ & $6 \mathrm{~b}$ \\
\hline Hexyl 2-'methylbutyrate & $873 \mathrm{a}$ & $477 \mathrm{bc}$ & $768 \mathrm{ab}$ & $156 \mathrm{~cd}$ & $46 \mathrm{~d}$ & $11 \mathrm{~d}$ \\
\hline Total methyl butyrate esters & 1775 & 1110 & 1652 & 339 & 138 & 18 \\
\hline Hexyl tiglate $^{x}$ & $10 \mathrm{a}$ & $2 b$ & $2 b$ & $1 \mathrm{~b}$ & $2 b$ & $1 \mathrm{~b}$ \\
\hline 6-Methyl-5-hepten-2-one ${ }^{\mathrm{x}}$ & $8 \mathrm{a}$ & $13 \mathrm{~b}$ & $10 \mathrm{~b}$ & $5 \mathrm{~b}$ & $3 \mathrm{~b}$ & $0 \mathrm{~b}$ \\
\hline 4-Allylanisole $\mathrm{e}^{\mathrm{x}}$ & $216 \mathrm{a}$ & $36 \mathrm{~b}$ & $48 \mathrm{~b}$ & $44 \mathrm{~b}$ & $25 \mathrm{~b}$ & $9 \mathrm{~b}$ \\
\hline 1-Butanol & $211 \mathrm{a}$ & $57 \mathrm{bc}$ & $120 \mathrm{~b}$ & $12 \mathrm{c}$ & $11 \mathrm{c}$ & $8 \mathrm{c}$ \\
\hline 1-Pentanol & $5 \mathrm{a}$ & $1 \mathrm{bc}$ & $4 a b$ & $2 \mathrm{bc}$ & $1 \mathrm{bc}$ & $1 \mathrm{c}$ \\
\hline 1-Hexanol & $88 \mathrm{a}$ & $28 \mathrm{~b}$ & $78 \mathrm{a}$ & $1 \mathrm{~b}$ & $6 \mathrm{~b}$ & $2 \mathrm{~b}$ \\
\hline 2 Methyl-1-butanol & $79 \mathrm{a}$ & $27 \mathrm{bc}$ & $48 \mathrm{~b}$ & $49 a b$ & $36 \mathrm{bc}$ & $11 \mathrm{c}$ \\
\hline Total alcohols & 383 & 115 & 250 & 79 & 54 & 22 \\
\hline
\end{tabular}

${ }^{\mathrm{z}} 16$ weeks in CA followed by 4 weeks in RA.

y'Mean separation within rows by Fisher's protected LSD, $P \leq 0.05$.

${ }^{\mathrm{x}}$ Odor-active compounds at those concentrations.

${ }^{\text {w}}$ Above the linear range of the FID.

peak areas of odors perceived by Osme. For volatile compounds, FA using the principal component method was performed on the data correlation matrix to account for differences in peak scaling (Johnson and Wichern, 1992). For each set of Osme peak intensity and peak area variables, FA using the principal component method was performed on the covariance matrix of the residuals of a general linear model (GLM) where panelist was the main effect. The GLM residuals were used to remove the variability due to panelists using different parts of the scale (Piggott and Sharman, 1986). The residuals covariance matrix was then used to maximize differences between storage treatments. Based on the eigenvalues and the scree tests for each analysis (Tabachnick and Fidel, 1989), two factors were extracted from the principal component initial analysis, and rotated with the Varimax method. 

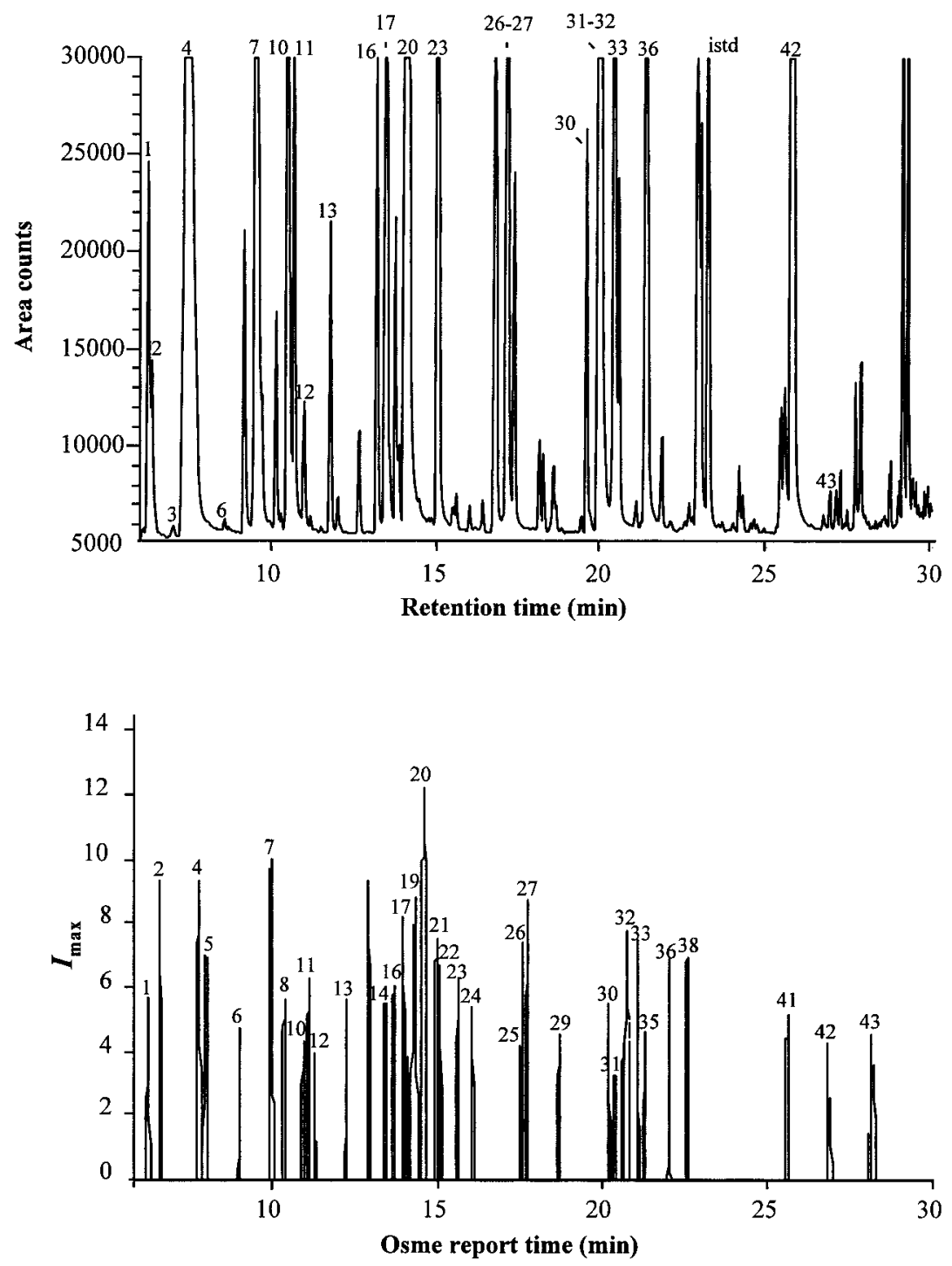

RA, and the amount increased again after 20 weeks in RA. The increase in volatile production from apples stored 10 to $60 \mathrm{~d}$ in RA has been reported previously (Brackmann and Streif, 1994). However, the higher amount of volatiles emitted after 20 weeks in RA is contradictory with other findings where less volatiles were produced after the longest time in RA (Fellman and Mattheis, 1995; Mattheis et al., 1998a; Streif and Bangerth, 1988). The same trend was observed from apples harvested the previous year (data not presented).

Total volatiles detected decreased during CA storage (Table 1). Volatile production by apples exposed to air for 4 weeks after 16 weeks in CA storage was greater than fruit stored 20 weeks in CA but production did not reach the level of apples stored 10 weeks in CA. A similar effect was reported in earlier work with 'Cox's Orange Pippin' (Smith, 1984), and 'Golden Delicious' (Streif and Bangerth, 1988).

Acetate esters comprised the largest proportion of volatiles emitted by 'Gala' apples; butyl acetate, hexyl acetate, and 2-methylbutyl acetate were produced in the largest amounts (Table 1). While all straight-chain esters showed a significant decrease in production after CA storage, 2-methylbutyl acetate was still produced in high amounts after 16 weeks in CA (Table 1). Similar results have been reported previously (Mattheis et al., 1998a). Both straight and branched-chain esters decreased under the concentrations of $\mathrm{O}_{2}$ and $\mathrm{CO}_{2}$ used in our study with the exceptions of 2-methylbutyl acetate, and its precursor 2-methyl-1-butanol (Table 1). Emission of straight-chain esters decreases more than branched-chain esters following storage in low $\mathrm{O}_{2}$ concentrations, and branched-chain esters decrease after storage in high $\mathrm{CO}_{2}$ concentrations (Brackmann et al., 1993).

The only ester emitted in higher amounts follow-

Fig. 1. Flame-ionization detector (FID) chromatogram (top) and Osme aromagram (bottom) for 'Gala' apples stored in air $\left(2{ }^{\circ} \mathrm{C}\right)$ for 4 weeks. Dynamic headspace samples were collected from $1-\mathrm{kg}$ apples for $24 \mathrm{~h}$ on charcoal traps. Only odoractive compounds are numbered. See Tables 2 and 3 for identity.

By rotating the first two factors, high and low correlations were maximized and minimized, respectively. This procedure is performed to clarify the importance of the linear combinations. The plots of storage treatments in the two-factor coordinate system allowed determination of the direction in which peak intensity (or peak area) variation pulled the storage factor scores. In other words, the graphical representation indicated the measurements (peak values) that best separated the data into storage treatment categories.

\section{Results and Discussion}

VOLATILE PRODUCTION AFTER STORAGE. IEC (mean $\pm \mathrm{SD}=2.45$ $\left.\mathrm{mL} \cdot \mathrm{L}^{-1} \pm 0.93\right)$ and starch scores (mean $\left.\pm \mathrm{SD}=2.4 \pm 0.5\right)$ at harvest were indicative of maturity acceptable for CA storage (Mattheis et al., 1998a). 'Gala' apples stored 4 weeks in RA emitted the largest amount of volatiles (Table 1). Then, a decrease in volatile production for most compounds was observed after 10 weeks in ing CA was 3-methyl-2-butenyl acetate (Table 1). This production pattern in response to CA storage has also been reported for 3-methyl-2-butenyl acetate produced by 'Jonagold' apples (Hansen et al., 1990).

Compounds other than esters detected included several alcohols, one allyl phenol, and one ketone. With the exception of 2-methyl-1-butanol, production of all of these compounds decreased after RA and CA storage for 10 weeks (Table 1).

VOLATILE PERCEPTION AFTER STORAGE. A typical FID chromatoCompounds with an odor activity are presented on the FID and Osme chromatograms with the same numbers, referred to in Tables 2 and 3. Peak aroma intensity gives an indication of compound potency in the samples (Da Silva et al., 1993) and peak area integrates the duration of perceived odor activity (Tables 2 and 3). Panelist variability was significant for most compounds. Regression curves relating perceived intensity or peak area to odorant concentrations showed differences in panelist sensitivities for some compounds, and different responses to increasing concentrations (Plotto, 1998). Nevertheless, panelists were consistent in rating concentration changes with storage treatments, resulting in little or no significant panelist by treatment interaction.

Compounds with fruity odors were esters, except for 6-megram and Osme aromagram for 'Gala' apple is presented in Fig. 1. 
thyl-5-heptene-2-one (Table 2). Decreased ester production following CA storage resulted in a decrease in perceived intensities of fruity peaks. Hexyl acetate, butyl acetate, and 2-methylbutyl acetate, emitted by 'Gala' apples in the largest amounts of all compounds detected, were also perceived with the highest intensities until fruit had been stored 10 weeks in CA (Table 2). 2Methylbutyl acetate had the highest perceived intensity of all fruity compounds throughout CA storage. Odor intensities for these three compounds as well as other esters decreased after 10 weeks in CA, but the decrease for hexyl acetate and butyl acetate was greater than for 2-methyl butylacetate. Methyl 2methylbutyrate, propyl 2-methylbutyrate, and ethyl 2methylbutyrate were still rated at or above 5.0 (slight to moderate) after 16 weeks in CA storage (Table 2). These three compounds along with hexyl acetate, butyl acetate, and 2-methylbutyl acetate were all rated above 1.5 (just detectable) after 20 weeks in CA, while the remaining esters were almost not perceived. The odor of 3-methyl-2-butenyl acetate was also perceived higher after CA

Table 2. Peak aroma intensity $\left(I_{\max }\right)$ in 'Gala' headspace after regular (RA) or controlled atmosphere (CA) storage by Osme analysis. Values on a 16-point intensity scale $(0=$ none and $16=$ extreme $)$ are means of four replicates for three panelists.

\begin{tabular}{|c|c|c|c|c|c|c|c|c|}
\hline \multirow{2}{*}{$\begin{array}{l}\text { Peak } \\
\text { no. }\end{array}$} & \multirow{2}{*}{$\begin{array}{l}\text { Retention } \\
\text { index }^{z}\end{array}$} & \multirow[b]{2}{*}{ Descriptor (compound) } & \multicolumn{3}{|c|}{ RA storage (weeks) } & \multicolumn{3}{|c|}{ CA storage (weeks) } \\
\hline & & & 4 & 10 & 20 & 10 & $16^{\mathrm{y}}$ & 20 \\
\hline 20 & 1028 & Gala, ripe, pear (hexyl acetate) & $11.4 \mathrm{a}^{\mathrm{x}}$ & $9.3 \mathrm{~b}$ & $10.6 \mathrm{ab}$ & $9.3 \mathrm{~b}$ & $4.8 \mathrm{c}$ & $3.9 \mathrm{c}$ \\
\hline 4 & 827 & Nail polish, gala (butyl acetate) & $10.3 \mathrm{a}$ & 8.9 a & $9.6 \mathrm{a}$ & $8.1 \mathrm{ab}$ & $5.2 \mathrm{c}$ & $1.7 \mathrm{c}$ \\
\hline 7 & 890 & Solvent, gala (2-methylbutyl acetate) & $11.0 \mathrm{a}$ & $8.6 \mathrm{c}$ & $8.4 \mathrm{~cd}$ & $10.4 \mathrm{ab}$ & $9.2 \mathrm{bc}$ & $6.9 \mathrm{~d}$ \\
\hline 2 & 787 & Sweet, fruity (methyl 2-methylbutyrate) & $8.9 \mathrm{a}$ & $8.0 \mathrm{a}$ & $7.9 \mathrm{a}$ & $7.3 \mathrm{a}$ & $7.7 \mathrm{a}$ & $1.2 \mathrm{~b}$ \\
\hline 13 & 959 & Very sweet, strawberry (propyl 2-methylbut.) & $6.8 \mathrm{a}$ & $6.8 \mathrm{a}$ & $5.7 \mathrm{a}$ & $6.5 \mathrm{a}$ & $5.7 \mathrm{a}$ & $2.3 \mathrm{~b}$ \\
\hline 6 & 863 & Sweet, strawberry (ethyl 2-methylbutyrate) & $6.2 \mathrm{ab}$ & $6.7 \mathrm{ab}$ & $7.4 \mathrm{a}$ & $6.1 \mathrm{ab}$ & $4.9 \mathrm{~b}$ & $2.7 \mathrm{c}$ \\
\hline 23 & 1056 & Fruity, apple (butyl 2-methylbutyrate) & $5.6 \mathrm{a}$ & $3.7 \mathrm{~b}$ & $5.3 \mathrm{a}$ & $2.6 \mathrm{~b}$ & $1.0 \mathrm{c}$ & $0.2 \mathrm{c}$ \\
\hline 36 & 1255 & Apple, grapefruit (hexyl 2-methylbutyrate) & $5.1 \mathrm{a}$ & $2.4 \mathrm{bc}$ & $3.8 \mathrm{ab}$ & $1.3 \mathrm{~cd}$ & $0.9 \mathrm{~cd}$ & $0.2 \mathrm{~d}$ \\
\hline $31+32$ & 1205 & Green apple (butyl hexanoate + hexyl but.) & $4.7 \mathrm{ab}$ & $2.8 \mathrm{~b}$ & $6.3 \mathrm{a}$ & $3.4 \mathrm{~b}$ & $0.0 \mathrm{c}$ & $0.0 \mathrm{c}$ \\
\hline 19 & 1018 & Apple and toast (unknown) & $7.0 \mathrm{a}$ & $3.0 \mathrm{~b}$ & $2.2 \mathrm{bc}$ & $2.0 \mathrm{bc}$ & $2.5 \mathrm{bc}$ & $0.3 \mathrm{c}$ \\
\hline 16 & 997 & Fruity, tape (6-methyl-5-hepten-2-one) & $3.3 \mathrm{a}$ & $1.0 \mathrm{~b}$ & $0.3 \mathrm{~b}$ & $0.3 \mathrm{~b}$ & $0.6 \mathrm{~b}$ & $0.5 \mathrm{~b}$ \\
\hline 18 & 1010 & Solvent, gala (unknown) & $3.1 \mathrm{a}$ & $0.4 \mathrm{~cd}$ & $3.1 \mathrm{a}$ & $1.7 \mathrm{~b}$ & $1.3 \mathrm{bc}$ & $0.0 \mathrm{~d}$ \\
\hline 27 & 1121 & Apple (hexyl propanoate) & $3.0 \mathrm{a}$ & $1.3 \mathrm{abc}$ & $2.0 \mathrm{ab}$ & $0.5 \mathrm{bc}$ & $0.0 \mathrm{c}$ & $0.0 \mathrm{c}$ \\
\hline 17 & 1009 & Rotten apple (butyl butyrate) & $2.3 \mathrm{ab}$ & $2.3 \mathrm{ab}$ & $3.0 \mathrm{a}$ & $1.3 \mathrm{bc}$ & $0.0 \mathrm{c}$ & $0.0 \mathrm{c}$ \\
\hline 44 & 1524 & Fruity (unknown) & $1.6 \mathrm{a}$ & $1.9 \mathrm{a}$ & $1.2 \mathrm{ab}$ & $0.7 \mathrm{ab}$ & $0.0 \mathrm{~b}$ & $0.0 \mathrm{~b}$ \\
\hline 10 & 921 & Fruity, apple (butyl propanoate) & $1.5 \mathrm{a}$ & $1.7 \mathrm{a}$ & $0.7 \mathrm{ab}$ & $0.0 \mathrm{~b}$ & $0.0 \mathrm{~b}$ & $0.0 \mathrm{~b}$ \\
\hline 3 & 813 & Fruity (propyl propanoate) & $0.0 \mathrm{~b}$ & $0.2 \mathrm{ab}$ & $0.8 \mathrm{ab}$ & $1.4 \mathrm{a}$ & $0.6 \mathrm{ab}$ & $0.4 \mathrm{ab}$ \\
\hline \multirow[t]{2}{*}{12} & 933 & Fruity, sweet (3-methyl 2-butenyl acetate) & $0.4 \mathrm{c}$ & $0.6 \mathrm{c}$ & $0.6 \mathrm{c}$ & $5.2 \mathrm{a}$ & $4.8 \mathrm{a}$ & $2.7 \mathrm{~b}$ \\
\hline & & Total fruity & 91.6 & 69.1 & 78.3 & 63.1 & 44.4 & 20.3 \\
\hline 43 & 1437 & Grape juice (b-damascenone) & $6.2 \mathrm{a}$ & $2.2 \mathrm{bc}$ & $2.4 \mathrm{~b}$ & $2.2 \mathrm{bc}$ & $1.4 \mathrm{c}$ & $0.0 \mathrm{~d}$ \\
\hline \multirow[t]{2}{*}{38} & 1276 & Grape juice (unknown) & $1.7 \mathrm{a}$ & $0.0 \mathrm{~b}$ & $0.5 \mathrm{~b}$ & $0.0 \mathrm{~b}$ & $0.0 \mathrm{~b}$ & $0.0 \mathrm{~b}$ \\
\hline & & Total grape juice & 7.9 & 2.2 & 2.9 & 2.2 & 1.4 & 0.0 \\
\hline 26 & 1118 & Floral (unknown) & $1.9 \mathrm{a}$ & $0.0 \mathrm{~b}$ & $0.0 \mathrm{~b}$ & $0.0 \mathrm{~b}$ & $0.0 \mathrm{~b}$ & $0.0 \mathrm{~b}$ \\
\hline 24 & 1070 & Watermelon (unknown) & $6.9 \mathrm{a}$ & $1.0 \mathrm{~b}$ & $0.0 \mathrm{c}$ & $0.0 \mathrm{c}$ & $0.0 \mathrm{c}$ & $0.1 \mathrm{c}$ \\
\hline \multirow[t]{2}{*}{34} & 1227 & Cucumber (unknown) & $2.2 \mathrm{a}$ & $0.4 \mathrm{~b}$ & $0.8 \mathrm{~b}$ & $0.0 \mathrm{~b}$ & $0.0 \mathrm{~b}$ & $0.0 \mathrm{~b}$ \\
\hline & & Total watermelon, cucumber & 9.1 & 1.4 & 0.8 & 0.0 & 0.0 & 0.1 \\
\hline 33 & 1222 & Anise, licorice (4-allylanisole) & $7.6 \mathrm{a}$ & $3.0 \mathrm{bc}$ & $2.3 \mathrm{bcd}$ & $3.5 \mathrm{~b}$ & $1.0 \mathrm{~cd}$ & $0.5 \mathrm{~cd}$ \\
\hline 22 & 1041 & Sweet, anise (unknown) & $1.3 \mathrm{ab}$ & $0.0 \mathrm{c}$ & $0.0 \mathrm{c}$ & $1.6 \mathrm{a}$ & $0.3 \mathrm{bc}$ & $0.0 \mathrm{c}$ \\
\hline \multirow[t]{2}{*}{29} & 1151 & Anise, spice, perfumey (unknown) & $2.6 \mathrm{a}$ & $0.6 \mathrm{bc}$ & $0.0 \mathrm{c}$ & $2.1 \mathrm{ab}$ & $0.4 \mathrm{c}$ & $0.9 \mathrm{bc}$ \\
\hline & & Total anise & 11.5 & 3.7 & 2.3 & 7.2 & 1.7 & 1.4 \\
\hline 35 & 1230 & Mushroom, cat urine (unknown) & $5.2 \mathrm{ab}$ & $3.1 \mathrm{~cd}$ & $6.9 \mathrm{a}$ & $4.6 \mathrm{bc}$ & $1.4 \mathrm{~d}$ & $1.4 \mathrm{~d}$ \\
\hline \multirow[t]{2}{*}{14} & 991 & Mushroom (1-octen-3-ol) & $2.9 \mathrm{a}$ & $0.3 \mathrm{~b}$ & $0.3 \mathrm{~b}$ & $0.0 \mathrm{~b}$ & $0.0 \mathrm{~b}$ & $0.0 \mathrm{~b}$ \\
\hline & & Total mushroom & 8.1 & 3.4 & 7.2 & 4.6 & 1.4 & 1.4 \\
\hline 5 & 836 & Skunk (unknown) & $9.0 \mathrm{a}$ & $4.0 \mathrm{~b}$ & $4.0 \mathrm{~b}$ & $5.2 \mathrm{~b}$ & $4.7 \mathrm{~b}$ & $3.4 \mathrm{~b}$ \\
\hline 25 & 1112 & Dusty, musty (unknown) & $6.4 \mathrm{a}$ & $5.3 \mathrm{a}$ & $3.8 \mathrm{a}$ & $4.3 \mathrm{a}$ & $2.4 \mathrm{~b}$ & $3.9 \mathrm{a}$ \\
\hline 37 & 1272 & Rubber (unknown) & $5.4 \mathrm{a}$ & $5.2 \mathrm{a}$ & $1.6 \mathrm{bc}$ & $2.0 \mathrm{~b}$ & $0.4 \mathrm{bc}$ & $0.0 \mathrm{c}$ \\
\hline 8 & 901 & Oatmeal, skunk (unknown) & 2.1 & 0.0 & 0.0 & 0.0 & 0.0 & 0.0 \\
\hline \multirow[t]{2}{*}{21} & 1038 & Metallic, skunk (unknown) & 1.6 & 0.0 & 0.0 & 0.0 & 0.0 & 0.0 \\
\hline & & Total skunk, rubber & 24.5 & 14.5 & 9.4 & 11.5 & 7.5 & 7.3 \\
\hline 1 & 774 & Tea, garlic, leaves (unknown) & 2.2 & 1.5 & 3.0 & 4.7 & 30 & 0.8 \\
\hline 30 & 1197 & Tape or fruity (unknown) & $1.7 \mathrm{a}$ & $0.0 \mathrm{~b}$ & $0.0 \mathrm{~b}$ & $0.2 \mathrm{~b}$ & $0.0 \mathrm{~b}$ & $0.7 \mathrm{~b}$ \\
\hline \multirow[t]{2}{*}{41} & 1364 & Tape or musty, dirty (unknown) & $1.6 \mathrm{~b}$ & $0.5 \mathrm{bc}$ & $3.3 \mathrm{a}$ & $0.3 \mathrm{c}$ & $1.1 \mathrm{bc}$ & $0.9 \mathrm{bc}$ \\
\hline & & Total tape, others & 5.5 & 2.1 & 6.4 & 5.3 & 4.1 & 2.4 \\
\hline 9 & 920 & Garlic (unknown) & $0.7 \mathrm{~b}$ & $0.0 \mathrm{~b}$ & $0.3 \mathrm{~b}$ & $3.5 \mathrm{a}$ & $3.0 \mathrm{a}$ & $1.2 \mathrm{~b}$ \\
\hline
\end{tabular}

${ }^{\mathrm{z}}$ Retention indices on RTX-5 (5\% diphenyl 95\% dimethyl polysiloxane) column.

y 16 weeks in CA followed by 4 weeks in RA.

${ }^{x}$ Mean separation within rows by Fisher's protected LSD, $P \leq 0.05$. 
storage (Table 2).

Other compounds with grape juice, floral, watermelon, cucumber, and anise odors, were perceived with a higher intensity after 4 weeks in RA (Table 2). B-Damascenone (2,6,6-trimethyl1-trans-crotonyl-1,3-cyclohexadiene) and 4-allylanisole (1methoxy-4-(2-propenyl)-benzene) were responsible for the most important of the two and three grape juice and anise odors, respectively, and were rated between 2.0 and 3.0 until 10 weeks in CA. Two mushroom-like peaks were perceived: peak 14, identified as 1-octen-3-ol by matching retention index and odor quality with a standard; and peak 35 , not identified. The total mushroom peak intensities were higher after 4 weeks in RA (Table 2).

Peaks with skunklike and rubberlike odors had higher ratings for apples stored 4 weeks in RA. These compounds had low odor thresholds as the chromatographic peaks were small or undetected by the FID, and remain unidentified. With these odor descrip-

Table 3. Perceived aroma peak area in 'Gala' headspace after regular (RA) or controlled atmosphere (CA) storage by Osme analysis. Values $\left(I_{\max }\right.$ $\times$ duration of odorant perception) are means of four replicates for three panelists.

\begin{tabular}{|c|c|c|c|c|c|c|c|c|}
\hline \multirow{2}{*}{$\begin{array}{l}\text { Peak } \\
\text { no. }\end{array}$} & \multirow{2}{*}{$\begin{array}{c}\text { Retention } \\
\text { index }^{z}\end{array}$} & \multirow[b]{2}{*}{ Descriptor (compound) } & \multicolumn{3}{|c|}{ RA storage (weeks) } & \multicolumn{3}{|c|}{ CA storage (weeks) } \\
\hline & & & 4 & 10 & 20 & 10 & $16^{\mathrm{y}}$ & 20 \\
\hline$\overline{20}$ & 1028 & Gala, ripe, pear (hexyl acetate) & $1.79 \mathrm{a}^{\mathrm{x}}$ & $0.99 \mathrm{bc}$ & $1.25 \mathrm{~b}$ & $0.85 \mathrm{c}$ & $0.35 \mathrm{~d}$ & $0.23 \mathrm{~d}$ \\
\hline 4 & 827 & Nail polish, gala (butyl acetate) & $1.70 \mathrm{a}$ & $0.94 \mathrm{c}$ & $1.25 \mathrm{~b}$ & $0.64 \mathrm{~d}$ & $0.29 \mathrm{e}$ & $0.06 \mathrm{e}$ \\
\hline 7 & 890 & Solvent, gala (2-methylbutyl acetate) & $1.26 \mathrm{a}$ & $0.65 \mathrm{~b}$ & $0.67 \mathrm{~b}$ & $1.03 \mathrm{a}$ & $0.67 \mathrm{~b}$ & $0.43 \mathrm{~b}$ \\
\hline 2 & 787 & Sweet, fruity (methyl 2-methylbutyrate) & $0.78 \mathrm{a}$ & $0.86 \mathrm{a}$ & $0.85 \mathrm{a}$ & $0.72 \mathrm{a}$ & $0.78 \mathrm{a}$ & $0.08 \mathrm{~b}$ \\
\hline 13 & 959 & Very sweet, strawberry (propyl 2-methylbut.) & $0.54 \mathrm{a}$ & $0.43 \mathrm{ab}$ & $0.42 \mathrm{ab}$ & $0.42 \mathrm{ab}$ & $0.33 \mathrm{~b}$ & $0.07 \mathrm{c}$ \\
\hline 6 & 863 & Sweet, strawberry (ethyl 2-methylbutyrate) & $0.47 \mathrm{~b}$ & $0.49 \mathrm{ab}$ & $0.69 \mathrm{a}$ & $0.43 \mathrm{~b}$ & $0.29 \mathrm{bc}$ & $0.12 \mathrm{c}$ \\
\hline 23 & 1056 & Fruity, apple (butyl 2-methylbutyrate) & $0.32 \mathrm{a}$ & $0.16 \mathrm{~b}$ & $0.32 \mathrm{a}$ & $0.12 \mathrm{bc}$ & $0.03 \mathrm{~cd}$ & $0.01 \mathrm{~d}$ \\
\hline 36 & 1255 & Apple, grapefruit (hexyl 2-methylbutyrate) & $0.32 \mathrm{a}$ & $0.10 \mathrm{bc}$ & $0.21 \mathrm{ab}$ & $0.07 \mathrm{c}$ & $0.04 \mathrm{c}$ & $0.01 \mathrm{c}$ \\
\hline $31+32$ & 1205 & Green apple (butyl hexanoate + hexyl but.) & $0.35 \mathrm{~b}$ & $0.17 \mathrm{c}$ & $0.51 \mathrm{a}$ & $0.18 \mathrm{c}$ & $0.00 \mathrm{~d}$ & $0.00 \mathrm{~d}$ \\
\hline 19 & 1018 & Apple and toast (unknown) & $0.78 \mathrm{a}$ & $0.24 \mathrm{~b}$ & $0.15 \mathrm{bc}$ & $0.15 \mathrm{bc}$ & $0.14 \mathrm{bc}$ & $0.01 \mathrm{c}$ \\
\hline 16 & 997 & Fruity, tape (6-methyl-5-hepten-2-one) & $0.17 \mathrm{a}$ & $0.03 \mathrm{~b}$ & $0.01 \mathrm{~b}$ & $0.02 \mathrm{~b}$ & $0.02 \mathrm{~b}$ & $0.01 \mathrm{~b}$ \\
\hline 18 & 1010 & Solvent, gala (unknown) & $0.17 \mathrm{a}$ & $0.02 \mathrm{~cd}$ & $0.13 \mathrm{ab}$ & $0.09 \mathrm{bc}$ & $0.04 \mathrm{~cd}$ & $0.00 \mathrm{~d}$ \\
\hline 27 & 1121 & Apple (hexyl propanoate) & $0.17 \mathrm{a}$ & $0.06 \mathrm{bc}$ & $0.10 \mathrm{ab}$ & $0.01 \mathrm{bc}$ & $0.00 \mathrm{c}$ & $0.00 \mathrm{c}$ \\
\hline 17 & 1009 & Rotten apple (butyl butyrate) & $0.08 \mathrm{a}$ & $0.11 \mathrm{a}$ & $0.13 \mathrm{a}$ & $0.06 \mathrm{ab}$ & $0.00 \mathrm{~b}$ & $0.00 \mathrm{~b}$ \\
\hline 44 & 1524 & Fruity (unknown) & $0.07 \mathrm{ab}$ & $0.10 \mathrm{a}$ & $0.03 \mathrm{ab}$ & $0.03 \mathrm{ab}$ & $0.00 \mathrm{~b}$ & $0.00 \mathrm{~b}$ \\
\hline 10 & 921 & Fruity, apple (butyl propanoate) & 0.05 & 0.05 & 0.04 & 0.00 & 0.00 & 0.00 \\
\hline 3 & 813 & Fruity (propyl propanoate) & 0.00 & 0.01 & 0.04 & 0.06 & 0.02 & 0.02 \\
\hline \multirow[t]{2}{*}{12} & 933 & Fruity, sweet (3-methyl 2-butenyl acetate) & $0.02 \mathrm{~b}$ & $0.02 \mathrm{~b}$ & $0.01 \mathrm{~b}$ & $0.31 \mathrm{a}$ & $0.25 \mathrm{a}$ & $0.12 \mathrm{~b}$ \\
\hline & & Total fruity & 9.01 & 5.40 & 6.80 & 4.86 & 2.99 & 1.05 \\
\hline 43 & 1437 & Grape juice (ß-damascenone) & $1.49 \mathrm{a}$ & $0.24 \mathrm{~b}$ & $0.30 \mathrm{~b}$ & $0.25 \mathrm{~b}$ & $0.11 \mathrm{~b}$ & $0.00 \mathrm{~b}$ \\
\hline \multirow[t]{2}{*}{38} & 1276 & Grape juice (unknown) & $0.08 \mathrm{a}$ & $0.00 \mathrm{~b}$ & $0.02 \mathrm{~b}$ & $0.00 \mathrm{~b}$ & $0.00 \mathrm{~b}$ & $0.00 \mathrm{~b}$ \\
\hline & & Total grape juice & 1.57 & 0.24 & 0.32 & 0.25 & 0.11 & 0.00 \\
\hline 26 & 1118 & Floral (unknown) & $0.09 \mathrm{a}$ & $0.00 \mathrm{~b}$ & $0.00 \mathrm{~b}$ & $0.00 \mathrm{~b}$ & $0.00 \mathrm{~b}$ & $0.00 \mathrm{~b}$ \\
\hline 24 & 1070 & Watermelon (unknown) & $0.48 \mathrm{a}$ & $0.04 \mathrm{~b}$ & $0.00 \mathrm{~b}$ & $0.00 \mathrm{~b}$ & $0.00 \mathrm{~b}$ & $0.00 \mathrm{~b}$ \\
\hline \multirow[t]{2}{*}{34} & 1227 & Cucumber (unknown) & 0.02 & 0.04 & 0.00 & 0.00 & 0.00 & 0.00 \\
\hline & & Total watermelon, cucumber & 0.50 & 0.08 & 0.00 & 0.00 & 0.00 & 0.00 \\
\hline 33 & 1222 & Anise, licorice (4-allylanisole) & $0.61 \mathrm{a}$ & $0.11 \mathrm{~b}$ & $0.10 \mathrm{~b}$ & $0.15 \mathrm{~b}$ & $0.03 \mathrm{~b}$ & $0.01 \mathrm{~b}$ \\
\hline 22 & 1041 & Sweet, anise (unknown) & $0.06 \mathrm{a}$ & $0.00 \mathrm{~b}$ & $0.00 \mathrm{~b}$ & $0.06 \mathrm{a}$ & $0.01 \mathrm{a}$ & $0.00 \mathrm{~b}$ \\
\hline \multirow[t]{2}{*}{29} & 1151 & Anise, spice, perfumey (unknown) & $0.12 \mathrm{a}$ & $0.02 \mathrm{ab}$ & $0.00 \mathrm{~b}$ & $0.12 \mathrm{a}$ & $0.02 \mathrm{ab}$ & $0.03 \mathrm{ab}$ \\
\hline & & Total anise & 0.79 & 0.13 & 0.10 & 0.34 & 0.07 & 0.04 \\
\hline 35 & 1230 & Mushroom, cat urine (unknown) & $0.35 \mathrm{~b}$ & $0.15 \mathrm{~cd}$ & $0.58 \mathrm{a}$ & $0.29 \mathrm{bc}$ & $0.07 \mathrm{~d}$ & $0.04 \mathrm{~d}$ \\
\hline \multirow[t]{2}{*}{14} & 991 & Mushroom (1-octen-3-ol) & $0.16 \mathrm{a}$ & $0.01 \mathrm{~b}$ & $0.02 \mathrm{~b}$ & $0.00 \mathrm{~b}$ & $0.00 \mathrm{~b}$ & $0.00 \mathrm{~b}$ \\
\hline & & Total mushroom & 0.51 & 0.16 & 0.60 & 0.29 & 0.07 & 0.04 \\
\hline 5 & 836 & Skunk (unknown) & $0.69 \mathrm{a}$ & $0.22 \mathrm{~b}$ & $0.23 \mathrm{~b}$ & $0.29 \mathrm{~b}$ & $0.28 \mathrm{~b}$ & $0.22 \mathrm{~b}$ \\
\hline 25 & 1112 & Dusty, musty (unknown) & $0.56 \mathrm{a}$ & $0.39 \mathrm{ab}$ & $0.27 \mathrm{~b}$ & $0.31 \mathrm{ab}$ & $0.12 \mathrm{~b}$ & $0.26 \mathrm{~b}$ \\
\hline 37 & 1272 & Rubber (unknown) & $0.51 \mathrm{a}$ & $0.24 \mathrm{~b}$ & $0.09 \mathrm{c}$ & $0.05 \mathrm{c}$ & $0.02 \mathrm{c}$ & $0.00 \mathrm{c}$ \\
\hline 8 & 901 & Oatmeal, skunk (unknown) & $0.08 \mathrm{a}$ & $0.00 \mathrm{~b}$ & $0.00 \mathrm{~b}$ & $0.00 \mathrm{~b}$ & $0.00 \mathrm{~b}$ & $0.00 \mathrm{~b}$ \\
\hline \multirow[t]{2}{*}{21} & 1038 & Metallic, skunk (unknown) & $0.06 \mathrm{a}$ & $0.00 \mathrm{~b}$ & $0.00 \mathrm{~b}$ & $0.00 \mathrm{~b}$ & $0.00 \mathrm{~b}$ & $0.00 \mathrm{~b}$ \\
\hline & & Total skunk, rubber & 1.90 & 0.85 & 0.59 & 0.64 & 0.42 & 0.48 \\
\hline 1 & 774 & Tea, garlic, leaves (unknown) & $0.15 \mathrm{bc}$ & $0.12 \mathrm{bc}$ & $0.23 \mathrm{ab}$ & $0.41 \mathrm{a}$ & $0.20 \mathrm{bc}$ & $0.04 \mathrm{c}$ \\
\hline 30 & 1197 & Tape or fruity (unknown) & $0.09 \mathrm{a}$ & $0.00 \mathrm{~b}$ & $0.00 \mathrm{~b}$ & $0.01 \mathrm{~b}$ & $0.00 \mathrm{~b}$ & $0.02 \mathrm{~b}$ \\
\hline \multirow[t]{2}{*}{41} & 1364 & Tape or musty, dirty (unknown) & $0.07 \mathrm{~b}$ & $0.01 \mathrm{c}$ & $0.18 \mathrm{a}$ & $0.01 \mathrm{c}$ & $0.03 \mathrm{bc}$ & $0.03 \mathrm{bc}$ \\
\hline & & Total tape, others & 0.30 & 0.13 & 0.42 & 0.44 & 0.23 & 0.09 \\
\hline 9 & 920 & Garlic (unknown) & $0.04 \mathrm{~b}$ & $0.00 \mathrm{~b}$ & $0.02 \mathrm{~b}$ & $0.21 \mathrm{a}$ & $0.18 \mathrm{a}$ & $0.07 \mathrm{~b}$ \\
\hline
\end{tabular}

Retention indices on RTX-5 (5\% diphenyl 95\% dimethyl polysiloxane) column.

y 16 weeks in CA followed by 4 weeks in RA.

${ }^{x}$ Mean separation within rows by Fisher's protected LSD, $P \leq 0.05$. 


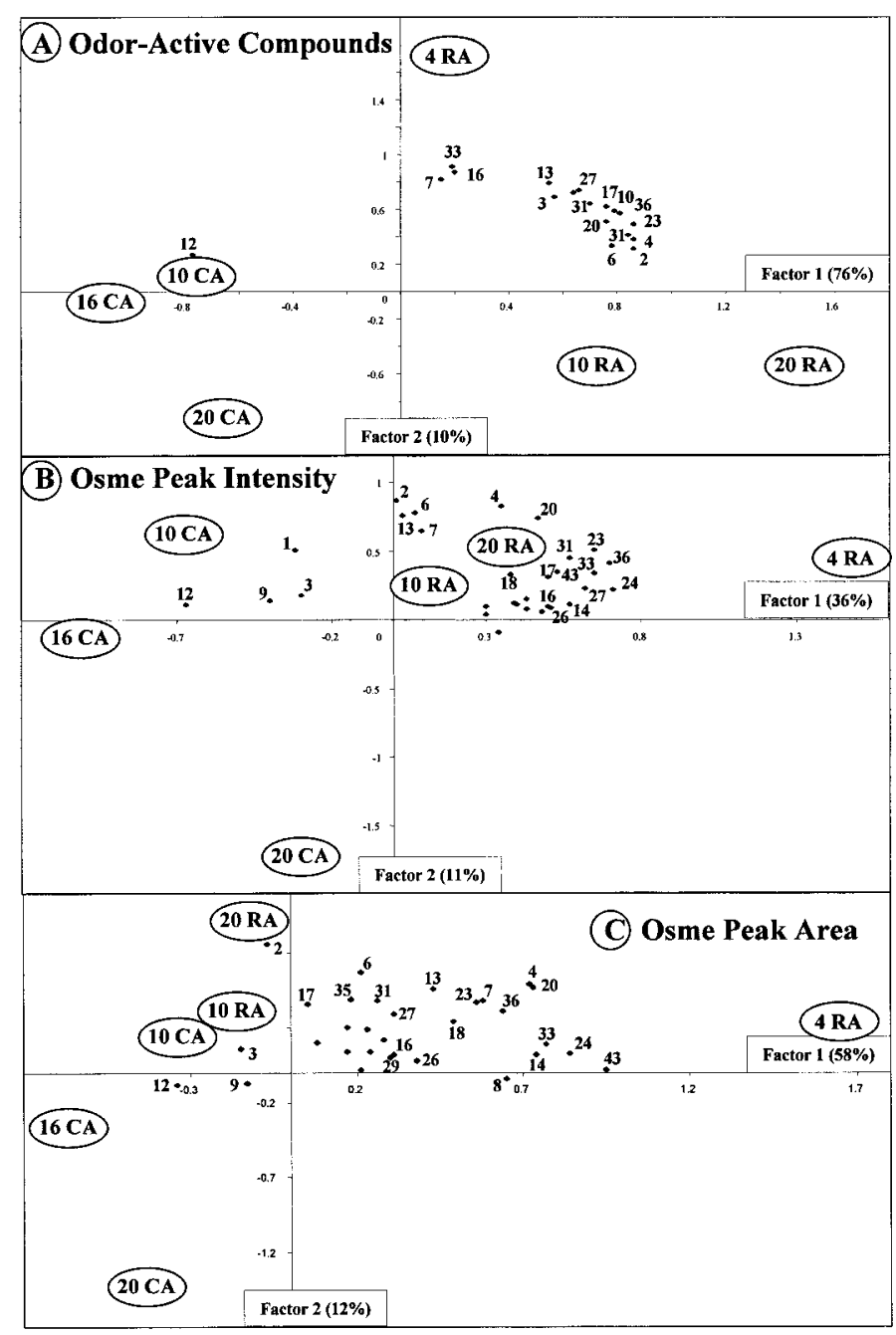

Fig. 2. (A) Factor plots of odor-active compound concentrations, (B) Osme peak intensity, and (C) Osme peak area of 'Gala' apples stored in regular (RA) and controlled atmosphere (CA) $\left(1 \mathrm{kPa} \mathrm{O}_{2}, 1 \mathrm{kPa} \mathrm{CO}\right)$. Scores for treatments are in the two-factor space. Four RA, 10 RA, 20 RA, 10 CA, and $20 \mathrm{CA}$ are 4, 10, and 20 weeks in RA and CA, respectively. Sixteen CA is 16 weeks in CA followed by 4 weeks in RA. Factor loadings were determined by the vector lengths for each peak. All vectors start at the origin and vector directions and magnitudes (loadings) are represented by the diamond symbols. Peak numbers: $1=$ unknown tea, leaves, $2=$ methyl 2 -methylbutyrate, $3=$ propyl propanoate, $4=$ butyl acetate, $6=$ ethyl 2-methylbutyrate, $7=2$-methylbutyl acetate, $8=$ unknown oatmeal, $9=$ unknown garlic, $10=$ butyl propanoate, $12=3$-methyl-2-butenyl acetate, 13 = propyl 2 -methylbutyrate, $14=1$-octen-3-ol, $16=6$-methyl-5hepten-2-one, $17=$ butyl butyrate, $18=$ unknown apple solvent, $20=$ hexyl acetate, 23 = butyl 2 -methylbutyrate, $24=$ unknown watermelon, $26=$ unknown floral, $27=$ hexyl propanoate, $29=$ unknown perfumey, anise, $31=$ butyl hexanoate + hexyl butyrate, $33=4$-allylanisole, $35=$ unknown mushroom, 36 $=$ hexyl 2-methylbutyrate, $43=$ B-damascenone. Diamond symbols without numbers in the $O$ sme factor plots represent odor-active peaks that contribute less significantly to the variation due to 'Gala' storage.

tors, the compounds may be nitrogen or sulfur-containing compounds such as 2-(methylthio)ethyl acetate, 3-methylthio-1-propanol, and 3-(methylthio)propyl acetate, reported previously by Schreier et al. (1978). Because of the high lability of sulfur compounds in stored samples (Hofmann et al., 1996) and their instability at each step of the GC run (Block, 1993), the identification and the representativeness of skunklike, rubberlike peaks perceived by Osme for 'Gala' apples remain to be proven. Descriptors such as rotten, putrid, earthy, mushroom, and dry dust were also reported from GCO of 'Golden Delicious' apples (Rizzolo et al., 1989). One compound with a garlic odor (peak 9) was perceived only from CA-stored fruit (Table 2).

Changes in peak areas across storage treatment were similar to those of peak intensities (Table 3). Discrimination between treatments was higher when measured with peak area for some compounds such as butyl acetate, but lower for other compounds such as 2-methylbutyl acetate. This suggests that each odoractive compound induces a different response to its concentration. Some compounds induce a fast response, resulting in a sharp peak, whereas others linger and produce a broad peak.

CORRELATIVE RELATIONSHIPS BETWEEN ODOR-ACTIVE VOLATILES. Factor analysis (FA) of odor-active compounds indicated factors 1 and 2 explained $76 \%$ and $10 \%$ of the total data set variation, respectively (Fig. 2A). The plots of factor scores reflected the decrease in all odor-active compounds during CA storage except 3-methyl-2-butenyl acetate (peak 12). Each peak is represented by its vector in the factor plots, vector angles reflect peak correlations with each other, and the vector magnitude [which is actually the variable (peak) loading] reflects the relative contribution of the peak to each factor. Fruit stored 20 weeks in RA had the highest factor 1 score followed by fruit stored 10 and 4 weeks in RA (Fig. 2A). The 4-week RA treatment had a high score for factor 2. Vectors for 2-methylbutyl acetate, 4-allylanisole, and 6-methyl-5-hepten-2-one had high loadings on factor 2 and were in the same direction as fruit stored 4 weeks in RA. This indicates these three compounds had the largest concentration difference between 4 weeks RA and the other storage treatments. All other esters explained the variation between RA and CAstored fruit; they had high loadings on factor 1, indicating higher concentrations in fruit stored 10 and 20 weeks in RA. The 10week and 16-week CA treatments, as well as the vector for 3methyl-2-butenyl acetate, had high negative scores for factor 1 and positive scores for factor 2 . The 20 -week CA treatment was located in the opposite quadrant from the FID peak vectors, indicating that fruit stored 20 weeks in CA produced the least amount of volatiles.

ODOR-ACTIVE PEAKS AND STORAGE TREATMENTS IN THE TWOFACTOR SPACE. The distribution of storage treatments in the space of the Osme peak intensities and peak areas reflect the higher ratings given to fruit from RA storage treatments. Factor 1 represented $36 \%$ and $58 \%$ of the variation in peak intensities and peak areas, respectively (Fig. 2B and C). The larger amount of variation explained by factor 1 for peak areas indicates larger covariation among peak areas than among peak intensity ratings. This could be the result of panelists being more consistent when rating peak area than peak intensity (Da Silva et al., 1994). Also, most of the variation expressed in factor 1 of peak area is due to the difference between 4-week RA and all other storage treatments. Factor 2 represented $11 \%$ and $12 \%$ of the variation in peak intensities and peak areas, respectively.

Apples stored 4 weeks in RA had a high factor 1 score in both peak intensity and peak area spaces. Vectors with high loadings on factor 1 in the peak intensity space were watermelon (peak 24), apple (butyl 2-methylbutyrate, peak 23), apple (hexyl 2methylbutyrate, peak 36), anise (4-allylanisole, peak 33), apple (hexyl propanoate, peak 27), and mushroom (peak 14) (Fig. 2B). Therefore, those compounds contributed most to the intensity of 'Gala' aroma-active compounds after 4 weeks in RA. Peaks due to methyl-, ethyl-, and propyl 2-methylbutyrate with sweet, fruity, berry odors (peaks 2, 6, and 13) and 2-methylbutyl acetate (solvent, fruity, peak 7) were correlated and had high loadings on 
factor 2. This indicates their contribution to apples stored in RA as well as in 10 weeks in CA. The vectors for hexyl acetate ('Gala', peak 20) and butyl acetate ('Gala', nail polish, peak 4) had high loadings on both factors 1 and 2, and were in the same directions as apples stored 10 and 20 weeks in RA. All RA treatments were in the positive quadrant; scores on factors 1 and 2 were lower for fruit stored 10 weeks in RA compared with fruit stored 4 and 20 weeks in RA, indicating lower ratings for peak intensity. The negative scores for CA treatments reflect the decrease in intensity ratings of odor-active peaks. The vectors for peak 9 (garlic), peak 12 (fruity, 3-methyl-2-butenyl acetate), peak 3 (apple, propyl propanoate) and peak 1 (tea, leaves) had negative scores for factor 1 , indicating higher intensity in CA storage (Fig. 2B).

The plot of factor scores for odor peak areas confirmed correlations between some of the peaks, and the difference between the 4week RA and other storage treatments was emphasized (Fig. 2C). Peak 43 (grape juice) had the highest score on factor 1, indicating the duration $\times$ intensity (peak area) of perception of $\beta$-damascenone was more important than intensity alone. B-Damascenone was previously reported to have a high Charm value, which indicates high intensity and duration of perception (Cunningham et al., 1986). By using Osme analysis, B-damascenone was perceived for a longer duration than other compounds possibly due in part to peak broadening during the GC analysis, and/or a different binding mechanism on the olfactory receptors. 4-Allylanisole (peak 33), watermelon (peak 24), and 1-octen-3-ol (mushroom, peak 14) also had high loadings on factor 1; perception of these compounds was highest after 4 weeks in RA (Tables 2 and 3). Similar to FA of peak intensities, hexyl acetate (peak 20) and butyl acetate (peak 4) were correlated and had high loadings on both factors. However, 2methylbutyl acetate (solvent, peak 7) was correlated with butyl 2methylbutyrate (apple, peak 23), hexyl 2-methylbutyrate (apple, peak 36), and propyl 2-methylbutyrate (sweet, berry, peak 13) (Fig. 2C). It appears there are different relationships between odor intensities and duration $\times$ intensity for these compounds. Unidentified peaks, peak 18 ('Gala', solvent), peak 26 (floral), peak 29 (anise, perfumey), and peak 16 (6-methyl-5-hepten-2-one, fruity), were also correlated to peak $23,7,36,4$, and 20 , but had lower loadings, indicating a lesser contribution to aroma of fruit stored 4 weeks in RA. Similar to peak intensities, methyl 2-methylbutyrate (peak 2) and ethyl 2-methylbutyrate (peak 6) had high loadings on factor 2 , both having a sweet fruity odor.

SignificAnCE OF ODOR ACTIVITIES AFTER STORAGE. Compounds perceived with higher intensities and duration $\times$ intensity from fruit stored 4 weeks in RA may contribute significantly to the characteristic aroma of fresh 'Gala' apple. Watermelon (unknown), 4allylanisole (anise), 1-octen-3-ol (mushroom), and B-damascenone (grape juice) on their own, do not have an apple-like odor. Their contribution to 'Gala' aroma may be by interactions with the other fruity, apple-like compounds in whole apples. A trained panel could perceive watermelon, anise, and cooked flavor (possibly due to ßdamascenone) in whole and cut 'Gala' apples (Plotto et al., 1999), at a low intensity. Anise aroma and the cooked flavor decreased in CA-stored apples. This is the first time that a watermelon-like odor is reported from apple aroma by GCO. The compound remains unidentified by the methods used in this study. 4-Allylanisole is also produced by 'Cox's Orange Pippin' and is responsible for the spicy character of that cultivar (Williams et al., 1977). Mushroom-like compounds are known to be emitted by 'Golden Delicious' apples (Rizzolo et al., 1989), and raspberry (Rubus ideaus L. 'Heritage') fruit (Roberts and Acree, 1996). B-Damascenone usually occurs in a glycosidically bound form and is present primarily in processed products due to hydrolysis of the glycosidic bond after crushing fruit cells (Roberts and Acree, 1995; Nursten and Woolfe, 1972). It has a very low odor threshold with a sweet, fruity, perfumey odor and is not typical of apple aroma in general. In spite of low volatility and its bound form, it was captured on charcoal traps from 'Gala' headspace during long sampling times.

Compounds contributing to the overall fruitiness of 'Gala' aroma in all RA treatments were hexyl acetate and butyl acetate described as ripe 'Gala' and 'Gala', nail polish, respectively, and butyl 2-methylbutyrate and hexyl 2-methylbutyrate, both described as apple, fruity. A positive correlation was observed between hexyl acetate and butyl acetate concentrations, perceived intensities, and peak areas, indicating production of these two compounds decreased similarly in response to CA conditions, and their olfactory perceptions were highly correlated with each other. The decrease in those compounds during CA-storage resulted in a loss of fruity flavor by DSA (Plotto et al., 1999). Hexyl acetate and butyl acetate were also identified as important compounds for 'Gala' aroma by comparing 'Gala' apples with model solutions (Plotto et al., 1998).

Peak intensity and peak area factor scores for methyl 2methylbutyrate, ethyl2-methylbutyrate, and propyl2-methylbutyrate indicate these compounds contributed to the aroma of fruit stored 20 weeks in RA. These compounds have distinctive sweet, fruity and strawberry, berrylike odors. Methyl 2-methylbutyrate and ethyl 2methylbutyrate have low odor thresholds: 0.25 and $0.1 \mu \mathrm{g} \cdot \mathrm{L}^{-1}$, respectively (Flath et al., 1967; Takeoka et al., 1989). This explains why these compounds are perceived from CA-stored apples even though they are emitted in very low amounts. These branched esters appear to be responsible for the underlying sweet and fruity aroma of 'Gala' apple (Plotto et al., 1999). 2-Methylbutyl acetate, with a nail polish odor, was also a significant 'Gala' odor volatile.

Other odor-active esters such as butyl and hexyl propanoate, butyl and hexyl butyrate, and hexyl hexanoate, as well as the ketone 6-methyl-5-hepten-2-one, had fruity, apple-like aromas. These compounds had lower intensities after RA storage, and usually were not perceived after CA storage (Table 2). They also had lower loadings in the FA, indicating they contributed less to the variability of odoractive peaks due to storage treatments.

The fruity peak corresponding to 3-methyl-2-butenyl acetate and the unknown garlic peak were only perceived after CA storage. The increase in 3-methyl-2-butenyl acetate after CA storage did not compensate for the loss of fruity aroma due to other esters (Plotto et al., 1999). The garlic peak was present at concentrations below the FID detection threshold and was not identified by GC-MS. This compound may contribute to a musty note found in CA-stored 'Gala' (Plotto et al., 1999).

This study confirmed the contribution of hexyl acetate, butyl acetate, and 2-methylbutyl acetate to 'Gala' aroma (Young et al., 1996). The GCO technique, Osme, allowed measurement of the odor activity of known and unknown volatile compounds in 'Gala' apple. The sensory analysis of GC effluents indicated what compounds were present in sufficient amounts to be odor-active regardless of detection by FID or MS. Because CA storage results in a loss of overall aroma volatile production, compounds contributing to fresh 'Gala' apple aroma were deduced by comparing fruit stored in CA and RA. Butyl 2-methylbutyrate and hexyl 2methylbutyrate contributed to the apple, fruity aroma in RAstored fruit. The lower molecular weight compounds methyl 2methylbutyrate, ethyl 2-methylbutyrate, and propyl 2methylbutyrate had stronger sweet and berrylike odors. 4Allylanisole, $\beta$-damascenone, 1-octen-3-ol, as well as the compound with a watermelon descriptor were important contributors 
of 'Gala' aroma after 4 weeks storage. Those results were obtained by using headspace sampling during a long period of time to capture as many compounds as possible. Aldehydes are also produced by 'Gala' apples (Mattheis et al., 1998b), but were not found by the sampling method used in this study. The odor activity of aldehydes in the 'Gala' apple matrix remains to be studied.

\section{Literature Cited}

Block, E. 1993. Flavor artifacts. J. Agr. Food Chem. 41:692.

Brackmann, A. and J. Streif. 1994. Ethylene, $\mathrm{CO}_{2}$ and aroma volatile production by apple cultivars. Acta Hort. 368:51-57.

Brackmann, A., J. Streif, and F. Bangerth. 1993. Relationship between a reduced aroma production and lipid metabolism of apples after long-term controlledatmosphere storage. J. Amer. Soc. Hort. Sci. 118:243-247.

Cunningham, D.G., T.E. Acree, J. Barnard, R.M. Butts, and P.A. Braell. 1986 Charm analysis of apple volatiles. Food Chem. 19:137-147.

Da Silva, M.A.A.P., V. Elder, C.L. Lederer, D.S. Lundahl, and M.R. McDaniel. 1993. Flavor properties and stability of a corn-based snack: Relating sensory, gaschromatography, and mass-spectrometry data, p. 707-738. In: G. Charalambous (ed.). Shelf life studies of foods and beverages. Elsevier, Amsterdam, The Netherlands.

Da Silva, M.A.A.P., D.S. Lundahl, and M.R. McDaniel. 1994. The capability and psychophysics of Osme: A new GC-olfactometry technique, p. 191-209. In: H. Maarse and D.G. Van Der Heij (eds.). Trends in flavour research. Elsevier, Amsterdam, The Netherlands.

Fellman, J.K. and J.P. Mattheis. 1995. Ester biosynthesis in relation to harvest maturity and controlled-atmosphere storage of apples, p. 149-162. In: R.L. Rouseff and M.M. Leahy (eds.). Fruit flavors: Biogenesis, characterization, and authentication. Amer. Chem. Soc. Symp. Ser. 596.

Fellman, J.K., D.S. Mattinson, B.C. Bostick, J.P. Mattheis, and M.E. Patterson. 1993. Ester biosynthesis in 'Rome' apples subjected to low-oxygen atmospheres. Postharvest Biol. Technol. 3:201-214.

Flath, R.A., D.R. Black, D.G. Guadagni, W.H. McFadden, and T.H. Schultz. 1967. Identification and organoleptic evaluation of compounds in 'Delicious' apple essence. J. Agr. Food Chem. 15:29-35.

Guadagni, D.G., S. Okano, R.G. Buttery, and H.K. Burr. 1966. Correlation of sensory and gas-liquid chromatographic measurements of apple volatiles. Food Technol. 20:166-169.

Hansen, K., L. Poll, and M.J. Lewis. 1990. The post-storage biosynthesis of flavour constituents in 'Jonagold' apples stored in different oxygen concentrations, $\mathrm{p}$. 129-132. In: Y. Bessière and A.F. Thomas (eds.). Flavor science and technology. Wiley, Chichester, U.K.

Hofmann, T., P. Schieberle, and W. Grosch. 1996. Model studies on the oxidative stability of odor-active thiols occurring in food flavors. J. Agr. Food Chem. 44:251-255.

Johnson, R.A. and D.W. Wichern. 1992. Applied multivariate statistical analysis. 3rd ed. Prentice Hall, Englewood Cliffs, N.J.

Mattheis, J.P., D.A. Buchanan, and J.K. Fellman. 1998a. Volatile compounds emitted by 'Gala' apples following dynamic atmosphere storage. J. Amer. Soc. Hort. Sci. 123:426-432.

Mattheis, J.P., D.A. Buchanan, and J.K. Fellman. 1998b. Volatiles emitted by 'Royal Gala' apples following sequential atmosphere storage. Acta Hort. 464:201206.

Mattheis, J.P., J.K. Fellman, P.M. Chen, and M.E Patterson. 1991. Changes in headspace volatiles during physiological development of 'Bisbee Delicious' apple fruit. J. Agr. Food Chem. 39:1902-1906.

McDaniel, M. R., R. Miranda-Lopez, B.T. Watson, N.J. Micheals, and L.M. Libbey. 1990. Pinot noir aroma: A sensory/gas chromatographic approach, p. 23-36. In: G. Charalambous (ed.). Flavors and off-flavors. Elsevier, Amsterdam, The Netherlands.

Nursten, H.E. and M.L. Woolfe. 1972. An examination of the volatile compounds present in cooked 'Bramley's Seedling' apples and the changes they undergo on processing. J. Sci. Food Agr. 23:803-822.

Patterson, B.D., S.G.S. Hatfield, and M. Knee. 1974. Residual effects of controlled atmosphere storage on the production of volatile compounds by two varieties of apples. J. Sci. Food Agr. 25:843-849.

Pérez, A.G., C. Sanz, J.J. Ríos, and J.M. Olías. 1993. Estudio comparativo de los perfiles aromáticos de manzana, plátano y fresa. (Comparison of main aroma constituents of apple, banana and strawberry.). Rev. Esp. Cienc. Tecnol. Aliment. 33:665-677.

Piggott, J.R. and K. Sharman. 1986. Methods to aid interpretation of multidimensional data, p. 181-232. In: J.R. Piggott (ed.). Statistical procedures in food research. Elsevier, London.

Plotto, A. 1998. Instrumental and sensory analysis of 'Gala' apple (Malus domestica Borkh.) aroma. PhD diss., Oregon State Univ., Corvallis.

Plotto, A., A.N. Azarenko, J.P. Mattheis, and M.R. McDaniel. 1995. 'Gala', 'Braeburn' and 'Fuji' apples: Maturity indices and quality after storage. Fruit Var. J. 49:133-142

Plotto, A., J.P. Mattheis, D.S. Lundahl, and M.R. McDaniel. 1998. Validation of gas chromatography olfactometry results for 'Gala' apple by evaluation of aromaactive compound mixtures, p. 290-302. In: C.J. Mussinan, and M.J. Morello (eds.). Flavor analysis: Developments in isolation and characterization. Amer. Chem. Soc. Symp. Ser. 705.

Plotto, A., M.R. McDaniel, and J.P. Mattheis. 1999. Characterization of 'Gala' apple aroma and flavor: Differences between controlled atmosphere and air storage. J. Amer. Soc. Hort. Sci. 124:416-423.

Rizzolo, A., A. Polesello, and S. Polesello. 1992. Use of headspace capillary GC to study the development of volatile compounds in fresh fruit. J. High Resolution Chrom. 15:472-476.

Rizzolo, A., A. Polesello, and G.Y. Teleky-Vàmossy. 1989. CGC/Sensory analysis of volatile compounds developed from ripening apple fruit. J. High Resolution Chrom. 12:824-827.

Roberts, D.D. and T.E. Acree. 1995. Developments in the isolation and characterization of B-damascenone precursors from apples, p. 190-199. In: R.L. Rouseff and M.M. Leahy (eds.). Fruit flavors: Biogenesis, characterization, and authentication. Amer. Chem. Soc. Symp. Ser. 596.

Roberts, D.D. and T.E. Acree. 1996. Effect of heating and cream addition on fresh raspberry aroma using a retronasal aroma simulator and gas chromatography olfactometry. J. Agr. Food Chem. 44:3919-3925.

SAS Institute, Inc. 1987. SAS/STAT guide for personal computers. version 6 ed. SAS Inst., Inc., Cary, N.C.

Schreier, P., F. Drawert, and M. Schmid. 1978. Changes in the composition of neutral volatile components during the production of apple brandy. J. Sci. Food. Agr. 29:728-736.

Smith, S.M. 1984. Improvement of aroma of 'Cox's Orange Pippin' apples stored in low oxygen atmospheres. J. Hort. Sci. 59:515-522.

Smock, R.C. 1979. CA storage of fruits. Hort. Rev. 1:301-336.

Song, J. and F. Bangerth. 1996. The effect of harvest date on aroma compound production from 'Golden Delicious' apple fruit and relationship to respiration and ethylene production. Postharvest Biol. Technol. 8:259-269.

Stebbins, R.L., A.A. Duncan, and C.O. Compton. 1994. Apple variety trial. 198894 progress report. Oregon State Univ. Ext. Serv., Corvallis.

Streif, J. and F. Bangerth. 1988. Production of volatile aroma substances by 'Golden Delicious' apple fruits after storage for various times in different $\mathrm{CO}_{2}$ and $\mathrm{O}_{2}$ concentrations. J. Hort. Sci. 63:193-199.

Tabachnick, B.G. and L.S. Fidel. 1989. Using multivariate statistics. 2nd ed. Harper Collins, New York.

Takeoka, G.R., R.G. Buttery, R.A.Flath, R.Teranishi,E.L. Wheeler, R.L. Wieczorek, and M. Guentert. 1989. Volatile constituents of pineapple (Ananas comosus [L.] Merr.), p. 223-237. In: R. Teranishi, R.G. Buttery, and F. Shahidi (eds.). Flavor chemistry: Trends and developments. Amer. Chem. Soc. Symp. Ser. 388.

White, A.G. 1991. The 'Gala' apple. Fruit Var. J. 45:2-3.

Wiley/NSB Database. PBM format, revision K.00.00. 1988. Agilent Technologies, Wilmington, Del.

Willaert, G.A., P.J. Dirinck, H.L. De Pooter, and N.N. Schamp. 1983. Objective measurement of aroma quality of 'Golden Delicious' apples as a function of controlled-atmosphere storage time. J. Agr. Food Chem. 31:809-813.

Williams, A.A., A.G.H. Lea, and C.F. Timberlake. 1977. Measurements of flavor quality in apples, apple juices, and fermented ciders, p. 71-88. In: R.A. Scanlan (ed.). Flavor quality objective measurement. Amer. Chem. Soc. Symp. Ser. 51.

Williams, M.W. and M.E Patterson. 1962. Internal atmosphere in 'Bartlett' pears stored in controlled atmospheres. Proc. Amer. Soc. Hort. Sci. 81:129-136.

Yahia, E., F.W. Liu, and T.E. Acree. 1990. Changes of some odor active volatiles in controlled atmosphere-stored apples. J. Food Qual. 13:185-202.

Yajima, I., T. Yanai, M. Nakamura, H. Sakakibara, and K. Hayashi. 1984. Volatile flavor components of 'Kogyoku' apples. Agr. Biol. Chem. 48:849-855.

Young, H., J.M. Gilbert, S.H. Murray, and R.D. Ball. 1996. Causal effects of aroma compounds on 'Royal Gala' apple flavours. J. Sci. Food Agr. 71:329-336. 